Bernard De Meyer

Bernard De Meyer est Professeur associé à l'Université du KwaZuluNatal (Pietermaritzburg). Co-éditeur de Changing Face of African Literature (Rodopi 2009), il a publié de nombreux articles sur les romanciers africains contemporains d'expression française.

E-mail:demeyerb@ukzn.ac.za

\section{L'hospitalité dans Le Roi de Kahel de Tierno Monénembo: de la construction identitaire peule à la consécration littéraire parisienne}

\section{Hospitality in Le Roi de Kahel (The King of Kahel) by Tierno Monénembo: from Pullo identity construction to literary consecration in Paris}

Taking the notion of hospitality, as defined by Émile Benveniste, as a starting point, this article aims to situate the 2008 novel by Tierno Monénembo, Le Roi de Kahel ("The King of Kahel”) in the contemporary literary production from Francophone Africa. In a first part, hospitality is viewed as part of an exchange system between the Pullo aristocracy and a French explorer, Olivier de Sanderval, in the second half of the nineteenth century. The notion of pulaaku, as the social and moral code of conduct of the Pullo, seems central in this exchange and is a construct of Pullo identity. The second part aims to show that Monénembo's novel enters in a dialogue with colonial writings and in particular with French ethnography of the twentieth century, being as such hospitable to this discourse. This "textual hospitality", through the quotation of several sources and the description of some notions, including pulaaku, is a way to become accepted in the Parisian literary system. The final part, which is also the conclusion, shows the reciprocity between both conceptions of hospitality. I will argue here that Tierno Monénembo, having a perfect understanding of the prevalent literary field, takes advantage of this knowledge to promote his own writing. This is the reason why he is one of the most prominent postcolonial writers, leading the way for this literature to become integral part of the world republic of letters, as defined by Pascale Casanova. Keywords: Tierno Monénembo, hospitality, identity construct, pulaaku, literary consecration.

Le neuvième roman de Tierno Monénembo, Le Roi de Kahel, paru en 2008, est devenu un succès de librairie et critique, ayant obtenu le Prix Renaudot en 2008. ${ }^{1} \mathrm{Il}$ me semble tout à fait pertinent de prendre comme angle d'approche pour l'analyse de cet ouvrage le concept d'hospitalité, me concentrant plus spécifiquement sur l'hospitalité dans un univers foncièrement hostile. ${ }^{2}$ La thématique du roman semble particulièrement bien s'insérer dans le sujet proposé, car il traite des efforts fournis durant la deuxième moitié du XIXe siècle par un certain Aimé Olivier, comte de Sanderval, de se tailler un royaume en terre hostile, celle des Peuls, au Fouta-Djalon, un massif montagneux dans l'arrière-pays de l'actuelle Guinée. Pour cela, il doit entrer en relation avec les autorités princières de ce peuple et se faire accepter par eux. Ce roman s'inspire de faits historiques, ainsi que de données biographiques et autobiographiques, en

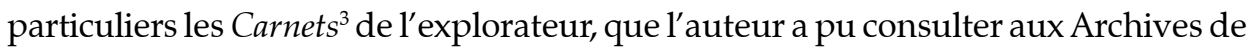
la ville du Caen, à 15 minutes de sa résidence actuelle. Dans un premier volet de cet 
article, j'indiquerai comment l'hospitalité, en tant que système d'échange, se trouve au centre des préoccupations de ce récit. Dans un deuxième volet, il sera question de l'accueil que l'auteur guinéen fait à la littérature coloniale et en particulier à l'ethnographie française, pour se faire accueillir, à son tour, par l'institution littéraire parisienne. Cette démarche mettra en valeur la réciprocité entre les deux acceptions de l'hospitalité. L'exposé se terminera avec quelques considérations sur la place de ce roman dans l'autonomisation progressive d'une littérature africaine d'expression française.

\section{Hospitalités}

Le terme hôte renvoie tout aussi bien à celui qui accueille qu'à celui qui est accueilli. Il serait donc judicieux de faire un petit tour sur l'origine de la polysémie de cette notion en français. Les deux significations, d'une part la "personne qui donne l'hospitalité" et d'autre part la "personne qui reçoit l'hospitalité" datent de la même période, la fin du douzième siècle, et ont la même origine, "du lat. hospitem, accusatif de hospes 'celui qui donne ou reçoit l'hospitalité'" (Robert 260-61). Le linguiste Émile Benveniste, dans une importante étude de 1969, remonte à la notion latine qui explique le double sens actuel en français; cette analyse est en outre un excellent point de départ pour l'étude que je vais mener ici. Sans reprendre toute l'argumentation de Benveniste, je me contenterai de montrer ici comment le roman de Tierno Monénembo joue sur cette notion somme toute ambigüe de "hôte". En effet, dans sa recherche sur l'étymologie, Benveniste note qu'

à la différence du peregrinus qui habite hors des limites du territoire, hostis est

l'étranger, en tant qu'on lui reconnait des droits égaux à ceux des citoyens romains.

Cette reconnaissance de droits implique un certain rapport de réciprocité et suppose une convention. N'est pas dit hostis quiconque n'est pas romain. Un lien d'égalité et de réciprocité est établi entre cet étranger et le citoyen de Rome, ce qui peut conduire à la notion précise d'hospitalité. (93-94)

N'est donc pas hôte qui le veut. Ceci est particulièrement vrai dans le contexte de la colonisation, où les forces coloniales ne recherchaient d'ordinaire pas l'hospitalité des peuples dans les terres qu'elles voulaient annexer. Dan ce sens, le projet sandervalien, tel que représenté par Monénembo, se trouve à contre-courant des pratiques de l'époque. Son désir de se faire accepter par les Peuls, d'être leur hôte, leur ami-les deux mots qui reviennent fréquemment dans le roman y sont interchangeables (hôte équivaut à ami et ami équivaut à hôte)—fait d'Aimé Olivier un original, lui donne l'envergure d'un personnage romanesque, lui donne droit de cité et d'être cité. Comme le souligne l'auteur lui-même dans une entrevue: "[Olivier de Sanderval] a ceci d'intéressant qu'il est un colonisateur solitaire et romantique, ce qui est une 
matière formidable pour un romancier" (Brezault 259). Sa position excentrique donne une importance particulière aux liens qu'il noue; commel'indique Abdoulaye Imorou, "le thème de l'amitié est des plus centraux dans le récit" (Imorou 8). Imorou souligne la "signification toute particulière" de ce thème: l'amitié "a valeur d'alliance. Elle renvoie à un pacte d'assistance et de non-agression" (8), qui n'est cependant jamais acquis.

Or, une des conditions pour qu'une vraie hospitalité puisse s'établir est un rapport d'égalité dans l'échange entre les deux parties. Olivier de Sanderval se distingue des autres explorateurs en voulant se faire accepter comme égal par les Peuls. Cette approche n'était pas évidente, d'abord à cause de l'éloignement géographique du Fouta-Djalon, ensuite à cause du comportement des Peuls, qui se font remarquer par leur caractéristiques exagérées et contradictoires: "tous les défauts et toutes les qualités de la terre", nous le rappelle Monénembo par son personnage: "radins, perfides, ombrageux; intelligents, raffinés, foncièrement nobles!" (Le Roi 112), "un peuple insondable, sublime et inquiétant" (145) que le narrateur remémore, de retour en France: "le monde mystérieux des Peuls—si sournois, si tordus, si nobles, si valeureux, si fascinants, en fin de compte, qu' on les paierait juste pour le prix de leurs défauts" (122). Ce dernier commentaire, de nature économique, montre que leur extrême originalité les rend digne d'un échange possible. Par ailleurs, le chemin qui mène vers eux, à travers l'océan et la brousse, est bien long et semé d'embuches. Chaque étape, que ce soit d'abord chez les autorités coloniales portugaises, anglaises et françaises, et ensuite parmi les peuples voisins du Fouta-Djalon et chez les seigneurs $\mathrm{du}$ territoire peule qu'il rencontre avant d'atteindre la capitale Timbo, où il va finalement rencontrer l'almâmi, le chef coutumier Peul, fait comprendre à Sanderval, au moment de l'accueil, qu'il doit s'adapter au comportement de ses hôtes.

En effet, le meilleur invité est celui qui imite parfaitement les attitudes de son hôte. Pour y parvenir, celui-là doit étudier les mœurs et coutumes du peuple et de la personne qui le reçoivent, aussi bien en aval, avant la réception, que sur le tas, par mimétisme. Plus l'imitation est réussie, plus l'accueil sera chaleureux et plus l'invité, qui vient d'habitude avec une requête, qu'elle soit exprimée ou non, obtiendra ce qu'il est allé chercher. Dans cette imitation, Sanderval accepte l'humanité des Peuls, et par retour il ne sera pas décapité, ce qui était le sort ordinaire des visiteurs peu prudents.

Car, et on le sait depuis la description du potlatch, festival et système d'échange économique parmi les populations indigènes de la côte nord-ouest atlantique des États-Unis et du Canada, par Marcel Mauss dans les années 1920 et 1930, la vraie hospitalité est fondée sur un échange, sur la triple obligation de donner, de recevoir et de rendre, "un des rocs humains sur lesquels sont bâties nos sociétés" (Mauss 27173). Le don n'est pas simplement une exhibition de l'hospitalité dans ce "jeu d'échange" (246), mais est gage de présent et d'avenir. Toutefois, son efficacité est la plus grande si les deux parties, le donateur et le receveur, utilisent un même système de valeur. Ceci 
n'est certainement pas le cas lors de la colonisation de l'Afrique du l'Ouest. D'une part, Olivier de Sanderval, capitaine de l'industrie dans son pays natal, qui a participé pleinement à la révolution industrielle en France (inventeur de la roue à moyeux suspendus, il a dirigé la première usine de vélocipèdes), est porteur d'un bagage capitaliste, privilégiant la mission civilisatrice de la colonisation. Il veut faire de son royaume dans le Fouta-Djalon une des provinces les plus évoluées en prenant en compte certaines valeurs occidentales, le modèle étant la Gaule romaine qui a su incorporer les valeurs de la Ville Eternelle. Devenu roi de Kahel, il y établira des factories et fera battre une monnaie, à son effigie, choses inconnues dans la tradition peule. D'autre part, au potlatch des indigènes nord-américains correspond le poulâkou chez les Peuls. A première vue il s'agit d'un système de règles de vie, une éthique semblable à celle décrite par Mauss, mais avec une nuance importante, comme le souligne un des personnages du roman, le roi de Kankalabé: “Nous sommes peuls, parents, notre éthique, le poulâkou, nous commande de nous conduire comme le caméléon: nous assurer que le monde ne va pas s'effondrer sous notre premier pas avant de risquer un second" (Monénembo, Le Roi 91). Ceci est pour le moins paradoxal, puisque le système de valeurs, comme ensemble de normes définissant le comportement, est dans ce cas foncièrement de nature changeante, s'adaptant aux situations telles qu' elles se présentent. Sanderval, malgré son caractère tempétueux, comprendra parfaitement cette façon d'agir et se comportera de façon conséquente; grâce à cette adaptation - il deviendra caméléon lui-même-il obtiendra la récompense ultime, celle qu'il brigue, la terre de Kahel. Dans cet échange, ce sont les préceptes de l'hospitalité et de l'amitié qui forment les seuls liens, comme le résume un autre Prince: "Nous sommes des Peuls, le poulâkou nous commande de bien traiter nos amis" (171). L'objectif de ces beaux principes est néanmoins d'établir un rapport de force et de montrer sa supériorité sur les invités.

Comme Benveniste l'avait déjà noté en discutant la notion plus large du don-et l'hospitalité serait la forme la plus élevée du don, don de sa demeure, don de soi-il existe une double responsabilité: à l'engagement de l'un (spondeo) répond la re-sponsio du second, qui induit la réciprocité du pacte en lui conférant une promesse (Benveniste 214 e.s.). Comme le résume Iñiguez, "I'hospitalité est une des manifestations les plus pleines du don de soi-même" (Iñiguez 41). La vraie hospitalité ne peut donc se faire par personne interposée. Ceci n'est décidément pas le cas dans la situation décrite dans le roman. L'almâmi ne s'adresse jamais directement aux rares personnes qu'il invite et passe pour tout échange par son griot. Le rôle de celui-ci dépasse la simple transmission des désirs de son chef. En effet, ce dernier ne s'exprime pas avec des paroles, mais avec des borborygmes et des grognements qui sont alors interprétés par l'intermédiaire officiel. Pour des raisons diverses, l'invité n'interpelle pas directement le chef coutumier; d'une part il n'en a pas le droit, et, d'autre part, en particulier lors de son premier voyage, il ne maitrise pas suffisamment bien la langue peule et doit se 
procurer des services d'un interprète, qui, Monénembo le rappelle dans son roman, est, avec le cuisinier, l'individu le plus important pour l'explorateur blanc (Monénembo, Le Roi 24, 28-29). La situation entre les deux camps est encore plus faussée par le fait que Sanderval doit s'assumer le rôle d'un personnage qu'il n'est pas, car les Princes peuls ne peuvent avoir affaire à un simple roturier; aussi prétend-il, sous les recommandations de son interprète, d'être le neveu du Roi de France-et on ne peut que noter l'incongruité et l' anachronisme de cette position en pleine Troisième République-et un déguisement de Méphistophélès, introduit subrepticement dans ses bagages par son épouse, a pu convaincre ses interlocuteurs ébahis (115). Tout ceci pour indiquer que les rapports entre les Peuls et Olivier de Sanderval, l'accueil de ce dernier par les habitants du Fouta Djalon, sont ancrées dans le faux, dans le leurre.

Bien qu'en marge de la brutalité coloniale, qui, ruinant l'économie des sociétés dites primitives, a dissous les systèmes sociaux ainsi que les équilibres souvent précaires entre les composantes d'une société et avec leurs voisins, les intentions de Sanderval y contribuent de manière significative, dans leur échec même. Le roman insiste plus sur les ratés du projet sandervalien, de ses fréquentes crises de paludisme aux empoisonnements alimentaires non moins récurrents, à son existence de paria, en particulier dans la jeune bourgade de Conakry à la fin du XIX ${ }^{e}$ siècle. Le désastre le plus important pour lui est la défaite militaire de l'armée peule dans la plaine de Porédaka en 1896 (235-36), car elle signale, avec la mort du dernier almâmi, Bôcar-Biro, non seulement la fin de l'indépendance du Fouta-Djalon, mais surtout l'impossibilité de réaliser son ambition. Le Roi de Kahel est un récit d'échecs: à l'échec de l'ambition personnelle d'Olivier de Sanderval, se joint celui, collectif, de l'Empire peul qui a atteint un stade de déclin inévitable. Ce sont les forces hostiles qui agissent dans l'ombre qui finissent par découper l'Afrique et par se l'approprier. Il n'est fait dans le roman qu'une très brève mention de la conférence de Berlin en 1885 (144), où la validité des traités de Sanderval ont néanmoins permis à la France de garder ce qui deviendra la Guinée française. ${ }^{4}$ Ces défaites représentent aussi l'échec, politique, au moins, de l'hospitalité, comme attitude ou même stratagème dans les rapports entre peuples à l'époque de la première colonisation. Toutefois, comme l'hospitalité est mise en valeur dans le roman, étant en fait le nœud de l'intrigue, il est nécessaire de voir ce que cela signifie.

\section{"Hospitalité du texte"}

On atteint ainsi le deuxième volet, qui seraitl'à rebours de ce premier parcours, mais fondé sur les mêmes principes, et nous propose de lire comment le roman de Tierno Monénembo intègre d'autres discours, en particulier les écrits coloniaux. Je renvoie ici à la notion de l'hospitalité du texte établie par Jean-Louis Cornille dans une analyse de Saint-Julien L'Hospitalier de Gustave Flaubert. Selon lui, un texte littéraire instaure 
un dialogue avec des ouvrages plus anciens; ces indices, sous forme de reprises, de jeux de mot, d'allusions, etc., incluent ce texte dans une ascendance qu'il revendique.

Un premier dialogue, très visible, et qui donne une voix à quelqu' un de l'autre bord, s'établit avec les écrits d'Olivier Sanderval lui-même, aussi bien ses ouvrages publiés - des récits de voyage au Fouta-Djalon qu'il a diffusés après son retour d'Afrique et un traité de philosophie autour de la notion de l'Absolu, sur lequel il a travaillé presque toute sa vie (Sanderval), que ses notes, en particulier les Carnets qu'il tenait lors de ses expéditions, et que Monénembo, jel'ai signalé, a épluchés aux archives de Caen. Le roman a été rédigé à partir de ces sources; Monénembo l'indique dans le paratexte (dans les remerciements) et, en outre, le récit contient une cinquantaine de citations entre guillemets, sans renvoi à la source exacte. Elles sont issues pour la plupart des carnets de l'explorateur. Ce qui rend ces extraits révélateurs, outre leur contenu, est qu'ils sont cités au moment même de leur rédaction, de la prise de note. Ils permettent au lecteur de littérature africaine, et à ceux qui s'intéressent à cette ère de l'histoire, de suivre l'idéologie en pénétrant à l'intérieur de cette période, qui est rarement représentée dans la littérature d'expression française rédigée par les habitants des anciennes colonies de la France, et certainement pour la première fois de façon si intense, exclusive, donnant même la voix à un colon.

Un deuxième dialogue est instauré grâce à un nombre assez conséquent de renvois qui est fait aux auteurs canonisés de la métropole, généralement dans des contextes qui soulignent la similitude. Je ne donnerai que trois exemples: décrivant Olivier de Sanderval, le narrateur conclut qu'"il avait bien la gueule de son époque. On s'imaginait Jules Verne, ou alors Victor Hugo" (37); le point commun étant bien sûr la barbe, mais le choix du récit se porte exclusivement sur des écrivains poilus, alors que d'autres Français de cette période, tel un Pasteur, se faisaient remarquer par leur impériale. Plus loin, une description des Peuls signalent que "ces gens sont insaisissables aussi bien par la main que par l'esprit! On dirait qu'ils ont tous lu Montaigne ici" (166). Parmi ces lecteurs de Montaigne se trouve évidemment Monénembo — et moins les Peuls analphabètes du XIXe siècle—en foi de quoi il est intégré dans la grande famille littéraire française. Ceci semble encore plus évident dans un écho balzacien, car, ayant "réussi à mettre un pied dans ce pays de tartufes et de brouillards", Sandarval s'écrie: "à nous deux, Fouta!" (107), en écho au défi que Rastignac lance à la société parisienne: "à nous deux maintenant!" (Balzac 1085). Cela dépasse la simple anecdote dans la mesure où Balzac avait rédigé La Recherche de l'absolu, un récit qui annonce certains aspects de la quête de l'Absolu chez Sanderval: un grand bourgeois qui délaisse sa famille au profit d'une cause plus grande.

Dans la même vaine, on pourrait s'interroger pourquoi parmi tous les déguisements possibles pour le héros qui doit faire face aux autorités peules, celui de Méphistophélès ait été choisi. Les lettres de noblesse littéraires de ce personnage diabolique ont été établies par Goethe. Il est clair que la thématique faustienne se retrouve dans le roman 
de Monénembo (Sanderval vend pour ainsi dire son âme dans son entreprise africaine). On ne peut par ailleurs que noter que l'auteur allemand se trouve à l'origine de la notion de Weltliteratur, ${ }^{5}$ qui a été remis au goût du jour et introduit dans l'univers de la francophonie par le manifeste de Michel Le Bris qui a proposé le concept de littérature-monde, au moment même où Monénembo rédigeait son récit. La publication de ce manifeste fut un des évènements littéraires principaux de cette année; elle remet en question les rapports qui existent entre les écrivains issus de la périphérie et le centre parisien, et est à l'origine d'un repositionnement fondamental du système littéraire français. Bien que Tierno Monénembo ne figure pas parmi les 44 auteurs signataires, il était conscient de l'apparition de ce manifeste, publié dans Le Monde en mars 2007, et dont les répercussions critiques et médiatiques ont été particulièrement larges. Il semble avoir intégré ce débat, par son personnage interposé, dans le roman qu'il préparait.

Olivier de Sanderval n'est ainsi pas seulement une figure historique et un personnage de roman, mais aussi un auteur et un lecteur. Il tient au fait le rôle d'ancêtre littéraire, car les premières lettres françaises de Guinée sont de sa main. Monénembo, en sa qualité d'auteur francophone originaire du Fouta Djalon revendique son ascendance et se place dans la lignée de celui qui le premier a écrit sur sa région natale en français, et qui était devenu Peul par adoption. De surcroit, et d'une façon qui pourrait paraître plus surprenante, la représentation du Peul dans ce roman se fait dans la tradition ethnologique qui remonte aux premières études occidentales sur les populations africaines, qui proviennent en fait d'Olivier de Sanderval lui-même! Aussi Le Roi de Kahel utilise-t-il la terminologie mise en place par l'ethnologie française de la première moitié du vingtième siècle, au lieu de l'orthographe scientifique moderne qui est basée sur les langues indigènes (ainsi, Monénembo utilise peul au lieu de pullo, peuls au lieu de fulbe, poulâkou au lieu de pulaaku). Cette façon d'orthographier les mots pourrait encore se comprendre-le roman rédigé en français s'adresse à un public francophone-, mais la notion du poulâkou, telle que définie par l'auteur guinéen, renvoie à cette même tradition.

Dans une étude extrêmement détaillée, Anneke Breedveld et Mirjam De Bruijn illustrent d'une part comment la recherche ethnologique utilise les stéréotypes racistes concernant les Peuls et d'autre part que la signification du poulâkou sur le terrain est extrêmement diversifiée. Ces deux chercheuses montrent que "l'origine moyenorientale et extra-africaine des Fulbe [...] semble motivée par un préjugé qui a ses racines dans les théories européennes du XIXe siècle sur les races" (Breedveld et De Bruijn 793). Monénembo reprend cette construction vraisemblablement fictive de l'identité peule et l'a développée dans son roman précédent, Peuls. L'écrivain se conforme aussi à la position que le poulâkou est un comportement social et moralselon Marguerite Dupire, dont l'interprétation, d'après Breedveld et De Bruijn qui la citent, a été reprise dans les recherches plus récentes, il s'agit plus prosaïquement de 
la "manière de se comporter en Peul" (cité par Breedveld et De Bruijn 795). Et aux deux chercheuses néerlandaises de conclure:

L'ensemble de ces études nous amènent à la conclusion que la notion de pulaaku est manipulée ou même inventée dans le discours politico-ethnique que les Fulbe tiennent sur eux-mêmes, et dans les relations qu'ils entretiennent avec le monde entier. A partir du moment où la notion de pulaaku fait son entrée dans la littérature scientifique consacrée aux Fulbe dans les années 1960, le terme a eu son propre dynamisme dans la construction de leur identité. (Breedveld et De Bruijn 799)

En conséquence, il y a une grande diversité d'interprétation, selon les régions, les groupes sociaux, ou encore parmi les hommes et les femmes, et qu'en fin de compte "ce mot ne signifie que l'ensemble de la communauté des Fulbe" (804).

Il est donc clair que la construction identitaire du Peul est un choix opéré par Tierno Monénembo, et s'est formée progressivement au cours de toute son œuvre. Ce choix n'est guère une représentation du quotidien vécu par la population, mais reflète une certaine conception du Peul qui s'est développée dans la pensée française, ou du moins dans la pensée en français. C'est donc en offrant l'hospitalité à cette pensée que l'œuvre de Monénembo est acceptée dans le système littéraire parisien: il est publié dans une maison d'édition renommée et Le Roi de Kahel a obtenu un des grands prix de la rentrée littéraire en 2008. Le personnage de Sanderval est essentiel dans cette production et l'image qu'il offre est composite. En tant que héros du roman, Olivier de Sanderval arrive à convaincre le lecteur, qui suit ses actions et ses pensées, du bien fondé de son comportement. Néanmoins, les citations tirées de ces carnets montrent une autre facette de l'explorateur, où il fait preuve d'une attitude condescendante envers les populations africaines, caractéristique de son époque. Ceci est exprimé, telle une mise en garde, dans une des épigraphes du roman : "Le Créateur les a faits noirs pour que les coups ne se voient pas.' (Olivier de Sanderval)" (Monénembo, Le Roi 11).

\section{Réciprocités}

Passons à l'étape finale de ce petit parcours. En accueillant la littérature coloniale dans ses propres récits, Monénembo, le seigneur de la demeure, s'attend à ce que Paris, comme centre d'où avait surgit le discours produit par cette littérature, lui rende des cadeaux. Aussi semble-t-il adapter la bravade de Rastignac et de Sanderval, qui devient pour lui: "A nous deux, Paris!" Le prix Renaudot, qui sera octroyé au roman en 2008, semble être la juste récompense; le voici accueilli à son tour dans l'antre de celui qu'il avait invité chez lui. On y lit bien sûr un élément d'annexion; le drame de l'écrivain francophone, selon Pascale Casanova, est que Paris est devenue une nécessité: 
La position des écrivains francophones est paradoxale, sinon tragique. Paris étant pour eux, inséparablement, la capitale de la domination politique et/ou littéraire et, comme pour tous les protagonistes de l'espace mondial, la capitale de la littérature, ils sont les seuls à ne pouvoir invoquer Paris comme tiers-lieu spécifique. Aucune alternative, aucune solution de rechange ne leur permet, en dehors d'un retrait dans leur espace national, [...] d'échapper à Paris ou de se servir de Paris pour inventer une dissidence esthétique. (Casanova 186)

Mais ce drame est également sa force: comme écrivain issu d'une contrée éloignée de la capitale littéraire, Monénembo fait preuve d'une grande lucidité et joue le jeu à merveille. Comme Sanderval, il devient un caméléon, il adapte son écriture pour se faire accepter, imitant avec talent des littératures qui se rapprochent de plus en plus du centre. Cette évolution se conjugue en plusieurs étapes. Le parcours a débuté chez lui avec le roman du désenchantement africain, façon d'entrer dans la maison Seuil qui a connu un énorme succès de librairie avec Les Soleils des indépendances d'Ahmadou Kourouma; il leur propose Les Crapauds-brousse en 1979, suivi par Les Écailles du ciel en 1986, qui obtient le Grand Prix de l'Afrique noire. Fort de cette récompense, il aborde les tendances dominantes, cherchant son inspiration dans le nouveau roman (pour Un rêve utile de 1991) et le roman latino-américain (dans le cas de Pelourinho, qui date de 1995), et enfin, une approche postcoloniale, qui semble faire l'unanimité aujourd'hui. L'écriture monénembéenne est cependant nourrie par la littérature coloniale, qui serait un des lieux de mémoire. En effet, la thématique qui domine dans ses derniers romans est la quête identitaire, dans un univers dominé par l'hybridité et l'altérité.

Comme pour Sanderval, la consécration de Monénembo se fait tarder. Après une entrée remarquée, le premier roman publié immédiatement dans une maison d'édition réputée, Le Seuil, le deuxième roman récompensé par le Grand Prix de l'Afrique Noire, il a dû attendre vingt-deux ans, un purgatoire qui était simultanément période de formation, et la publication de sept romans, dont quatre qui ne sont jamais parus en format de poche, avant de décrocher un des grands prix littéraires de la rentrée. Le retour aux avant-scènes s'est fait grâce à sa participation à un évènement littéraire et médiatique, le festival "Rwanda: écrire par devoir de mémoire" qui a eu lieu quatre ans après le génocide et qui est à l'origine de son roman L'Aîné des orphelins.

Les évènements du Rwanda, comme le soulignent des penseurs tels Achille Mbembe ou encore Patrice Nganang, ont fortement influencé la pensée et la littérature africaines d'après 1994, qui, pour ce dernier, est "nécessairement post-génocide" (Nganang 27). La confrontation aux horreurs du présent a encouragé la diversification thématique et stylistique parmi les écrivains du continent. Moins ouvertement politiques-entre l'impossibilité de décrire l'éminemment politique et la relative futilité des conflits locaux ou nationaux—et se distanciant simultanément des situations 
personnelles, les romans du XXI siècle semblent faire écho à la "diversalité" préconisée par les auteurs caribéens, 20 ans plus tôt. Ce processus d'autoréflexion montre la maturité de certains écrivains dans ce champ et a précipité son insertion dans la République mondiale des lettres (Casanova), combinant un humanisme inédit avec des innovations stylistiques. Les écrivains de l'exil en particulier sont libérés des préoccupations politiques immédiates, et, pour la majorité d'entre eux, ils sont à la recherche d'une définition plus pure de la littérature. Monénembo, dans son style bien à lui, présente un nouvel humanisme, une ontologie retorse dans ce qu'Homi Bhabha appelle un tiers espace d'énonciations, qui, comme l'explique ce dernier, "ouvre éventuellement la voie à la conception d'une culture internationale, fondée [...] sur l'inscription et l'articulation de l'hybridité de la culture" (83). Les jeux sur la narration et les renvois aux canons littéraires font preuve d'originalité, mais aussi d'une connaissance profonde du système littéraire qui a pour centre Paris. Monénembo, et je terminerai là-dessus, représente de façon exemplaire cette nouvelle génération d'écrivains africains qui affirment leur volonté de dépasser la nation, ces auteurs excentrés qui acceptent l'hospitalité offerte par l'institution littéraire. Le Roi de Kahel, comme tout roman qui innove, met en abyme les procédés qui sous-tendent son élaboration, ce qui garantit son accueil dans la République Mondiale des lettres.

\section{Notes}

1. Un "sacre mérité" selon Boniface Mongo-Mboussa, et Le Monde salue cette "biographie romancée foisonnante, épique et savoureuse [qui] sort de l'oubli cet africaniste avant la lettre" (Rousseau).

2. Cet article est une adaptation d'une communication présentée à la troisième conférence internationale du département de langues et littératures modernes, Université du Witwatersrand, Johannesburg, intitulée Hospitalité et hostilité dans le village multilinguistique mondial, qui s'est tenue du 12 au 14 septembre 2011. Il suit également une autre étude sur le même roman, étude qui analyse le lien entre le récit et la colonisation (De Meyer).

3. Ces Carnets n'ont pas été édités et sont consultables aux archives de Caen, en France. C'est toutefois en se servant de ces notes que Sanderval publie ses récits de voyage, tels De l'Atlantique au Niger par le Foutah-Djallon, carnet de voyage (1879-1880) (Paris: Ducrocq, 1882) et Soudan français. Kahel, carnet de voyage (Paris: Felix Alcan éditeur, 1893).

4. "Ses rapports avec le ministère de la Marine étaient toujours aussi froids, mais en 1885, à la conférence de Berlin, la France fut obligée de le reconnaître negotiorum gestor et de se revendiquer de ses traités pour faire échec aux Anglais." (Monénembo, Le Roi 144)

5. Le néologisme apparait chez Goethe à partir de 1827, d'abord dans son journal intime, ensuite dans des articles et dans sa correspondance. Voir Strich 397 sqq.

\section{Références}

Balzac, Honoré de. Le Père Goriot. La Comédie humaine. Volume 1. Paris: Pléiade, 1976.

—. La Recherche de l'absolu. Paris: Gallimard (Folio), 1976.

Benveniste, Émile. Le Vocabulaire des institutions indo-européennes. Volume 1. Paris: Minuit, 1969.

Bhabha, Homi. Les Lieux de la culture. Une théorie postcoloniale. Paris: Éditions Payot \& Rivages, 2007 [The Location of Culture. London, NY: Routledge, 1994].

Breedveld, Anneke, et De Bruijn, Mirjam. "ĹImage des Fulbe: analyse critique de la construction du concept du pulaaku." Cahiers d'études africaines, 36.144 (2010): 791-821. 
Brezault, Éloïse. Afrique, paroles d'écrivains. Montréal: Éditions mémoire d'encrier, 2010.

Casanova, Pascale. La République mondiale des lettres. Paris: Éditions du Seuil, 2008 [1999].

Cornille, Jean-Louis. "L'Hospitalité du texte." French Studies in Southern Africa, 37 (2007): 1-22.

De Meyer, Bernard. "La Colonisation selon Sanderval: Le Roi de Kahel de Tierno Monénembo." French Studies in Southern Africa, 31 (2011): 60-79.

Imorou, Adboulaye. "LÉtranger, le frère, l'ami: variations autour du roi de Kahel." InterFrancophonies, 3 (2010): 1-13.

Iñiguez, Huberto Giannini. "Hospitalité et tolérance." Philosophica, 66.2 (2000): 35-43.

Kourouma, Ahmadou. Les Soleils des Indépendances. Paris: Éditions du Seuil. 1970 [1968].

Le Bris, Michel, et al. "Pour une 'littérature-monde' en français." Le Monde des livres, 16 mars 2007.

Mauss, Marcel. Sociologie et anthropologie. Paris: Presses Universitaires de France, 1985 [1950].

Monénembo, Tierno. L'Aîné des orphelins. Paris: Éditions du Seuil, 2000.

—. Les Crapauds-brousse. Paris: Éditions du Seuil, 1979.

—. Pelourinho. Paris: Éditions du Seuil, 1995.

—. Peuls. Paris: Éditions du Seuil, 2004.

—. Le Roi de Kahel. Paris: Éditions du Seuil, 2008

—. Un rêve utile. Paris: Éditions du Seuil, 1991.

Mongo-Mboussa, Boniface. "Le sacre de Tierno Monénembo." Africultures, 10 nov. 2008. 3 juin 2012. $<$ http://www.africultures.com/php/index.php?nav =article\&no=8176>.

Robert, Paul, et al. Le Grand Robert de la langue française. Tome V (Grim-Lil). Paris: Le Robert, 1989.

Nganang, Patrice. Manifeste d'une nouvelle littérature africaine. Paris: Homnisphère, 2007.

Rousseau, Christine. "Le Prix Renaudot attribué à Tierno Monénembo." Le Monde, 12 nov. 2008. 3 juin 2012. <http://www.lemonde.fr/livres/article/2008/11/10/le-prix-renaudot-attribue-a-tiernomonenembo_1117068_3260.html\#ens_id=1114200>.

Sanderval, Olivier Aimé. De l'absolu. Loi de la vie. Paris: Félix Alcan éditeur, 1887.

Strich, Fritz. Goethe und die Weltliteratur. Bern: A. Francke, 1946. 